\title{
Shape of Moving Grain Boundary and its Influence on Grain Boundary Motion in Zinc
}

\author{
Vera Sursaeva ${ }^{a}$, Boris Straumal ${ }^{b}$ \\ Institute of Solid State Physics, Russian Academy of Sciences \\ Chernogolovka, Moscow District, RU-142432, Russia \\ asursaeva@issp.ac.ru bstraumal@issp.ac.ru
}

Keywords: grain boundary migration, grain boundary shape, grain boundary faceting, zinc

\begin{abstract}
The migration of individual special $[10 \overline{1} 0]$ tilt grain boundary (GB) with $\Sigma=15$ and misorientation angle $29^{\circ}$ in $\mathrm{Zn}$ bicrystal have been investigated. The stationary shape of migrating GB has been studied and the migration rate has been measured by optical microscopy in situ between 558 and $683 \mathrm{~K}$ using polarized light. In certain experimental runs the migrating GB is faceted and moves thermally activated. Its kinetics follows the Arrhenius type dependence despite the fact that shape of moving GB depends on temperature. After detachment from impurity cloud the $[10 \overline{1} 0]$ tilt GB migrates activationless in the temperature interval $618 \div 683 \mathrm{~K}$. The detachment temperature is $618 \mathrm{~K}$. The non-physically high value of the apparent migration activation enthalpy can appear due to the change of GB shape from faceted to smooth and back.
\end{abstract}

\section{Introduction}

The classical concepts of grain growth in polycrystal are based on a dominant role of grain boundaries. This is reflected by the well known von Neumann-Mullins relation. The classical von Neumann-Mullins relation of two-dimensional grain growth kinetics [1, 2], determines the change rate of the grain area

$$
\dot{S}=-A_{b}\left(2 \pi-\frac{n \pi}{3}\right)=\frac{A_{b} \pi}{3}(n-6),
$$

where $A_{b} \equiv m_{b} \gamma_{b}$ is the reduced GB mobility, $m_{\mathrm{b}}$ is GB mobility, $\gamma_{\mathrm{b}}$ is GB surface tension, $n$ is the number of triple junctions for the respective grain, i.e. the topological class of the grain. Accordingly, the rate of area change is independent on the shape of GBs and is determined by the topological class $n$ only. In this contribution, we focus on conclusion from Eq.1, which needs to be proved experimentally.

GB motion during grain growth is controlled by the GB curvature, which in turn is determined by the GB shape. The shape of a moving GB is a source of very useful data with regard to

- The orientation dependence of GB surface tension and GB mobility;

- Interaction between a moving GB and different kinds of obstacles.

There are important reasons why we consider the problem of the shape of a moving GB so comprehensively:

- All theories of GB motion assume planar GBs, while real GBs are curved;

- All changes associated with the joint motion of grain boundary and impurity cloud, the detachment of GB from the impurity cloud, and the motion of the free curved GB and faceted GB are clearly reflected in the shape of moving GB. 
Despite its importance, there has been only few research dedicated to the shape of moving GBs [3-5]. The problem of the shape of a moving GB was first considered by Mullins [1]. His equations provide a basis for all studies in this area.

The GB shape is defined by thermodynamic and kinetic conditions. From the thermodynamic point of view it is possible to discuss the equilibrium shape of GBs under external limitations imposed on the system. The equilibrium shape of GB is created by the flattening of different faces (planes); in doing so, the larger are crystallographic indices of the plane, the smaller is its area. This means that the equilibrium shape of GB consists of a small number of flat sections with a low energy, which are connected by curved parts, where all crystallographic planes are represented.

The shape of GB would be smooth as long as $\gamma_{b}$ varies continuously with GB inclination $\theta(\theta$ is the angle between the normal to GB element and direction of motion). If, however, it changes discontinuously with $\theta$, the faceting of moving GB would occur.

In case of impurity drag some parts of the GB may move freely, while the slower moving section is loaded with impurities. The vertex of the GB half-loop (Fig. 1) moves fast, and its velocity is equal to the velocity $V$ of the half-loop as a whole. The velocity $v_{\mathrm{F}}$ describes the velocity of a free GB (i.e. GB which is broken-away from the impurity atmosphere). The velocity $v_{\mathrm{L}}$ is determined by the diffusion of the impurities together with the moving GB. At intermediate $v$ values a transition from GB loaded with impurities to the broken-away GB occurs. In the transition regime GB would also move either as loaded or as a free GB. At some point GB can change from one state to another, as confirmed experimentally. $v^{*}$ is the critical value of GB velocity where the impurities can still move together with the half-loop GB:

$$
v^{*}=V \sin \left(\frac{\pi}{2} \frac{V_{F}-V}{V_{F}-V_{L}}\right)
$$

If the velocity $v$ exceeds $v^{*}$, the GB consists both of free segments (detached from impurities) as well as a loaded segments (moving together with the impurities). The GB segments which are far from the half-loop vertex move relatively slowly and, therefore, do not detach from the impurities [6].

We suppose that the kinetic conditions define the shape of moving GBs more than thermodynamic conditions. It is difficult to decide, whether the shape is defined by GB roughening or by the breakaway effects. We suppose that GB faceting and breakaway of GB from adsorbed impurities are closely connected. It is possible to imagine the situations when the velocities for breakaway and faceting are equal, but in other cases the GB velocities are different, and we can observe breakaway or faceting. The purpose of the paper is to assess the role of the boundary shape in GB motion.

\section{Experimental}

Only bicrystal techniques permit us to obtain reliable and reproducible data on GB mobility. It is possible to grow bicrystals with half loop GB geometry (Fig. 1), where the size of a shrinking crystal is lower than $0.2 \mathrm{~mm}$. This allows one to obtain relatively large driving forces for break away from impurity cloud and relatively small velocity for faceting. We use this technique due to its reproducibility and the feasibility to control the magnitude of the driving force, which is provided by surface tension of a GB. The observation of GB motion by orientation contrast is convenient and reliable, and does not only yield the location of a moving GB, but also its shape at any moment. In Figs. 6 and 8 individual video frames of GB motion in zinc bicrystals are given as observed by polarized light in an optical microscope. 
The bicrystalline samples were grown by a modified Bridgman technique in a high purity argon atmosphere in a graphite crucible. The crystallography and geometry of a bicrystal ( $\mathrm{Zn} \mathrm{99.999at} \mathrm{\% )} \mathrm{is} \mathrm{shown} \mathrm{in} \mathrm{Fig.} \mathrm{1.} \mathrm{GB} \mathrm{is} \mathrm{a} \mathrm{special}$ $[10 \overline{1} 0]$ tilt GB with reverse density of coincidence sites $\Sigma=15$ and angle $29^{\circ}$. It is known that this GB possesses high GB velocity at elevated temperature. This velocity is enough for break away from impurity cloud [7-9]. We observe also the faceting of moving GBs at elevated temperatures.

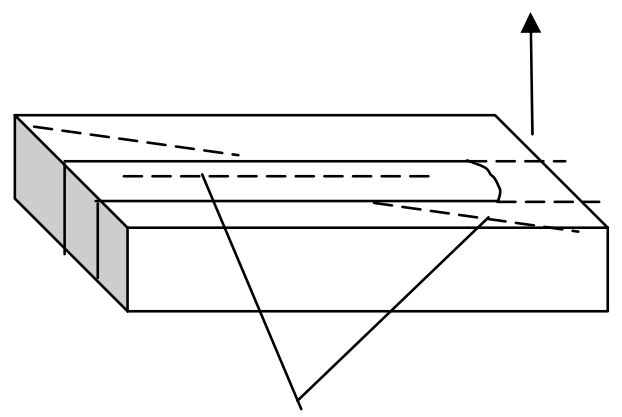

Basal planes in neighbor grains

Fig. 1. The scheme for the "half loop method".

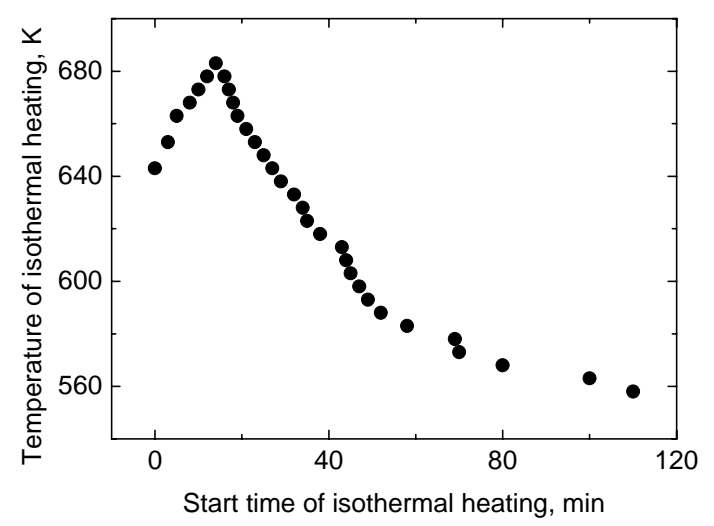

Fig. 2. Sequence of isothermal annealings during mobility studies.

To study the GBs migration in-situ at elevated temperatures a modified optical microscope with polarised light and a hot stage was used. It is possible to determine the mobility of system from the time dependence of GB displacement and a shape of moving GB at any moment (Figs. 3 to 5). The orientations of plane sample and the adjacent grains in bricrystals were determined by an investigation of the fracture surface of cracked sample. For the latter method small cracks were induced in the sample cooled by liquid nitrogen by a sharp knife. The cracks propagated along the basal plane of each grain. Hence, the misorientation could be determined by the angle between basal planes in neighboring grains, and plane orientation could be determined by the angle between basal plane and GB plane. The experiments were carried out according to scheme of Fig. 2 in the temperature range 558 to $683 \mathrm{~K}$.

\section{Results and discussion}

We analyzed the shape of GBs simultaneously with time dependence of displacement of half loop vertex. Time period of heating at elevated temperatures was changed as a rule between $120 \div 180 \mathrm{~s}$. It was found experimentally that this time is enough to observe the changing of displacement and shape of GB. In certain experimental runs the facet can form in $\theta=29^{\circ}[10 \overline{1} 0]$ tilt GB. In other experimental runs $\theta=29^{\circ}[10 \overline{1} 0]$ tilt GB does not facet and remains rough in the same temperature interval. The micrographs obtained in situ in hot stage using polarized light show typical shapes of GB, namely with facet in GB with $\theta=29^{\circ}$ (Fig. 6) and without facet (Fig. 8).

Different time dependences for displacement of special $29^{\circ}[10 \overline{1} 0$ tilt GB in $\mathrm{Zn}$ are presented in Figs. 3 to 5: curved (Fig. 3), straight (Fig. 4), straight sections with different slopes (Fig. 5). Curved and straight dependences corresponds to the moving GBs with facets (Fig. 6), straight sections with different slopes correspond to the motion with and without adsorbed impurity (Fig. 8). It is confirmed by changing GB shape. The breakaway flattens GB shape as theory predicted [6]. An experimental support of this viewpoint is the hysteresis of the transition temperature. On 


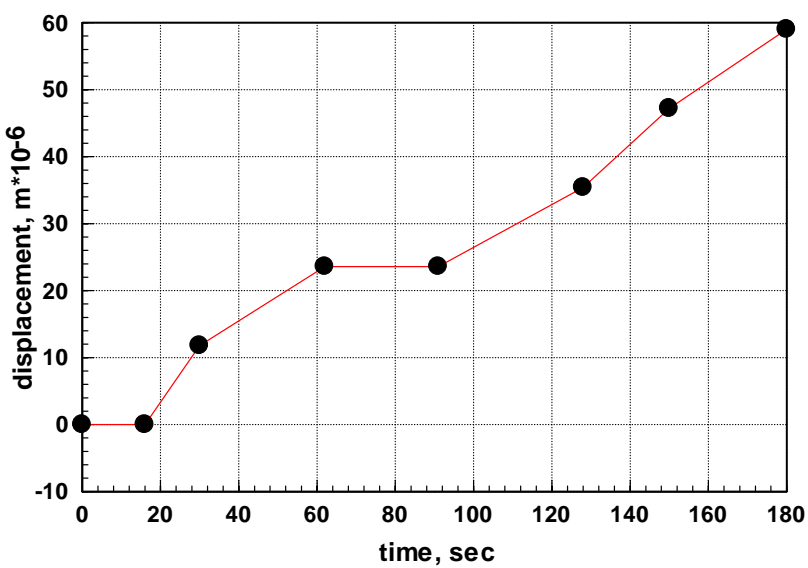

Fig. 3. Time dependence of GB displacement at $643 \mathrm{~K}$.

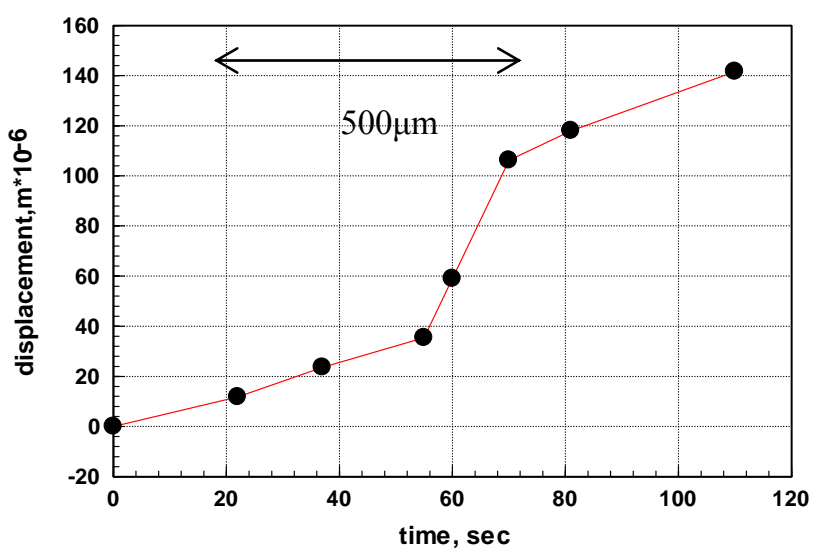

Fig. 5. Time dependence of GB displacement at $673 \mathrm{~K}$.

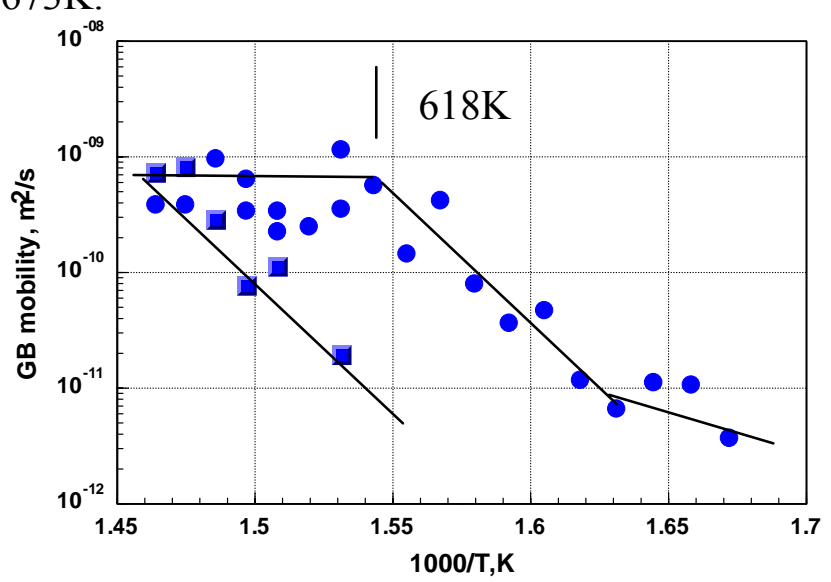

Fig.7. Temperature dependence of reduced GB mobility.

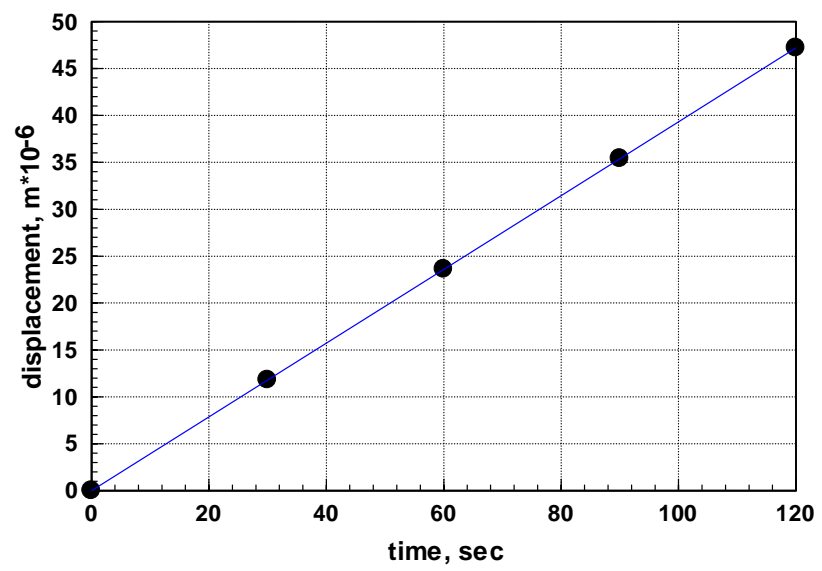

Fig. 4. Time dependence of GB displacement at $668 \mathrm{~K}$.

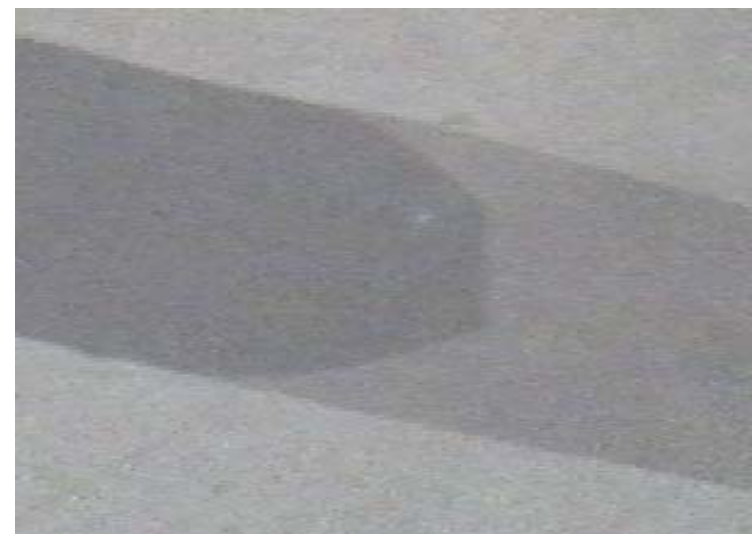

Fig. 6. Individual video frame of faceting GB motion

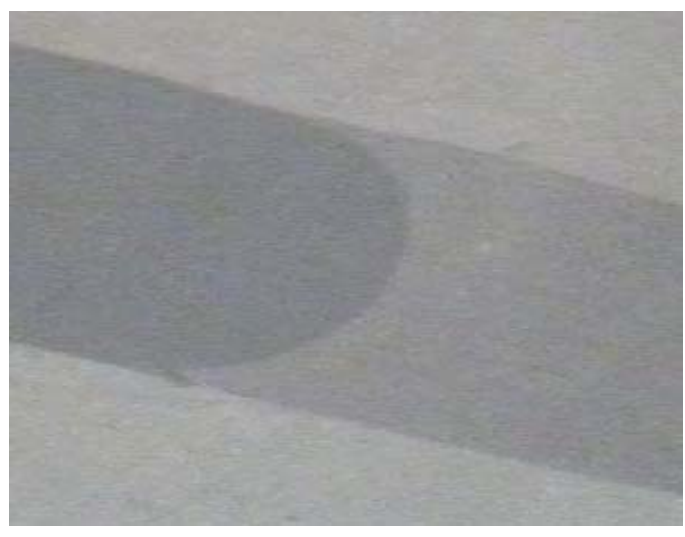

Fig. 8. Individual video frame of moving GB after breakaway from impurity cloud.

heating, the breakaway of a GB from an impurity cloud occurs at a temperature $(T=673 \mathrm{~K})$ higher than the re-attachment temperature of the GB impurities by cooling ( $T=618 \mathrm{~K})$ (Fig. 7). Despite the change of the GB shape we suppose that the driving force is provided by reduction of GBs energy and reads (per unit area): 


$$
p=\frac{2 \gamma}{a},
$$

where $\gamma$ is the surface tension of GB and $a$ is the width of the shrinking grain. The mobility of GB is given by the ratio of velocity $v$ and driving force $p$ :

$$
m=\frac{v}{p}=\frac{v a}{2 \gamma} .
$$

In our experiments the reduced mobility $A$ was used

$$
A=\frac{v a}{2}=m \gamma=A_{0} \exp \left(-\frac{H}{k t}\right),
$$

where $H$ is the activation enthalpy and $A_{O}$ is the pre-exponential factor.

Fig. 7 shows the temperature dependence of the reduced mobility of special $29^{\circ}[10 \overline{1} 0\rfloor$ tilt GB. Experimental data correlated well with predictions from the impurity drag theory $[12,13]$. The temperature dependence exhibits three sections:

- isothermal heating under increasing temperature, break away from impurity cloud at $T=$ $683 \mathrm{~K}$,

- isothermal heating under decreasing temperature, athermal motion of free $\mathrm{GB}$ at $683 \div 618 \mathrm{~K}$,

- isothermal heating under decreasing temperature, loaded GB motion after $618 \mathrm{~K}$.

The athermal motion after break away was observed during motion of special $[11 \overline{2} 0\rfloor$ tilt GBs only $[10,11]$. We observe the athermal motion of special $[10 \overline{1} 0]$ tilt GB for the first time.

\section{Concluding remarks}

- The migration of individual GB special $[10 \overline{1} 0]$ tilt GB with $\Sigma=15$ and misorientation angle $29^{\circ}$ in $\mathrm{Zn}$ bicrystal have been investigated.

- The stationary shape of the migrating GB has been studied and the migration rate has been measured in situ between 558 and $683 \mathrm{~K}$ using polarized light. In certain experimental runs the facet can form in the $\theta=29^{\circ}[10 \overline{1} 0]$ tilt GB.

- The migration of $[10 \overline{1} 0]$ tilt GB with misorientation angle $\theta=29^{\circ}$ is a thermally activated process. Its kinetics follows an Arrhenius type dependence despite the fact that moving GB shape is changed.

- For the first time it was found that $[10 \overline{1} 0]$ tilt GB manifest activationless motion after detachment from impurity cloud at $618 \div 683 \mathrm{~K}$ despite the fact that moving GB shape is changed. The temperature of detachment is $618 \mathrm{~K}$.

- The non-physically high value of the apparent migration activation enthalpy can appear due to the changing geometry of GB from faceted to smooth, similar to the case of migration of faceted twin tips [14].

\section{Acknowledgements}

These investigations were partly supported by Russian Foundation for Basic Research (contracts 03-02-04000 and 05-02-16528) and INTAS (contract 03-51-3779). 


\section{References}

[1] W.W. Mullins: J. Appl. Phys. Vol. 27 (1956), p. 900

[2] J. Von Neumann: in Metal Interfaces (American Society for Testing Materials, Cleveland 1952), p. 108

[3] A.V.Antonov, Ch.V.Kopetskii, L.S.Shvindlerman and Ya.M. Mukovskii: phys. stat. sol. (a) Vol. 9 (1972), p. 45

[4] V.Yu. Aristov, V.E. Fradkov and L.S. Shvindlerman: Sov. Phys. Solid State Vol. 22 (1980), p. 1055

[5] J.Ch. Verhasselt, G. Gottstein, D.A. Molodov and L.S. Shvindlerman: Acta Mater. Vol. 47 (1999), p. 887

[6] G. Gottstein and L.S. Shvindlerman: Grain Boundary Migration in Metals. Thermodynamics, Kinetics, Applications (CRC Press, Boca Raton 1999)

[7] V.Yu. Aristov, Ch.V. Kopeckii, V.G. Sursaeva and L.S. Shvindlerman: Sov. Phys. Dokl. Vol. 20 (1975), p. 842

[8] Ch.V. Kopeckii, V.G. Sursaeva and L.S. Shvindlerman: Scripta Metall. Vol. 12 (1978), p. 953

[9] Ch.V. Kopeckii, V.G. Sursaeva and L.S. Shvindlerman: Sov. Phys. Dokl. Vol. 23 (1978), p. 137

[10] Ch.V. Kopetskiy, L.S. Shvindlerman and V.G. Sursaeva: Scr. Metall. Vol. 12 (1978), p. 953

[11] Ch.V. Kopetskiy, V.G. Sursaeva and L.S. Shvindlerman: Sov. Phys. Solid State Vol. 21(1979), p. 238

[12] K. Lücke, K. Detert: Acta Metall. Vol. 5 (1957), p. 628

[13] K. Lücke and H. Stüwe: Acta Metall. Vol. 19 (1971), p. 87

[14] B.B. Straumal, E. Rabkin, V.G. Sursaeva and A.S. Gornakova: Zt. Metallkd. Vol. 96 (2005), p. 161 